\title{
BMJ open Micrographia and related deficits in Parkinson's disease: a cross-sectional study
}

\author{
Aparna Wagle Shukla, ${ }^{1}$ Songthip Ounpraseuth, ${ }^{2}$ Michael S Okun, ${ }^{1}$ Vickie Gray, ${ }^{3}$ \\ John Schwankhaus, ${ }^{3}$ Walter Steven Metzer ${ }^{3}$
}

To cite: Wagle Shukla A, Ounpraseuth S, Okun MS, et al. Micrographia and related deficits in Parkinson's disease: a cross-sectional study. BMJ Open 2012;2: e000628. doi:10.1136/ bmjopen-2011-000628

- Prepublication history for this paper is available online. To view these files please visit the journal online (http:// dx.doi.org/10.1136/ bmjopen-2011-000628).

Received 5 December 2011 Accepted 6 March 2012

This final article is available for use under the terms of the Creative Commons Attribution Non-Commercial 2.0 Licence; see http://bmjopen.bmj.com

${ }^{1}$ Department of Neurology, University of Florida, Center for Movement Disorders and Neurorestoration, Gainesville, Florida, USA

${ }^{2}$ Department of Biostatistics, University of Arkansas for Medical Sciences, Little Rock, Arkansas, USA ${ }^{3}$ Department of Neurology, University of Arkansas for Medical Sciences and the Central Arkansas Veterans Healthcare System (CAVHS), Little Rock, Arkansas, USA

Correspondence to Dr Aparna Wagle Shukla; aparna.shukla@neurology. ufl.edu

\section{ABSTRACT}

Objectives: To determine the prevalence and clinical features associated with micrographia in Parkinson's Disease (PD).

Setting: This study was conducted at a Movement Disorders clinic located in a Veteran Administration Hospital.

Participants: PD subjects were included only if they satisfied UK Parkinson's Disease Society criteria for diagnosis. Subjects with history of severe tremors, dystonia, dyskinesia, strokes, peripheral neuropathy and dementia were excluded.

Design: This was a case-control study where PD subjects were prospectively enrolled and their demographics, Hoehn \& Yahr stage, Unified Parkinson's Disease Rating Scale and Mini Mental Status examination (MMSE) scores were recorded. All subjects were specifically asked for micrographia on history and the handwritings were quantitatively documented. Bradykinesia was determined by history and quantified by a finger tap, Purdue pegboard and a timed walk test. Similarly, hypophonia was determined by history and the volume of speech quantified using a decibel meter. Controls were enrolled for validation of handwriting test scores and decibel meter recordings.

Primary outcome measures: Prevalence of micrographia in the PD cohort and the clinical factors that correlate with micrographia.

Results: 68 subjects with PD were enrolled (68 men; mean age 72.3 years). Micrographia was identified in $63.2 \%$ of the cohort on verbal history and in $50 \%$ of the cohort when the handwriting test was used for ascertainment. Micrographia ascertained on history correlated significantly with disease severity (Hoehn \& Yahr stage), motor impairment (Unified Parkinson's Disease Rating Scale), cognitive impairment (MMSE) and both bradykinesia and hypophonia determined by history and quantitative testing. Micrographia on handwriting test correlated with age $(p=0.02)$, MMSE testing $(p=0.04)$, hypophonia by history $(p=0.01)$ and bradykinesia by quantitative testing $(p=0.04)$.

Conclusion: Micrographia was found in nearly half of the PD cohort. Disease severity and impaired cognition were important clinical correlates. Micrographia had a significant relationship with bradykinesia and hypophonia, suggesting a possible overlap in their pathophysiology.

\section{ARTICLE SUMMARY}

Article focus

- In this study, prevalence of micrographia in Parkinson's disease (PD) is ascertained and the relationship of micrographia with bradykinesia and hypophonia is determined using standardized and quantitative assessment tools.

Key messages

- Micrographia is present in nearly $50 \%-60 \%$ PD cohort and disease severity and impaired cognition are important correlates.

- It has significant relationship with bradykinesia and hypophonia.

Strengths and limitations of this study

- Large sample size, systematic assessment methods.

- This study is a cross-sectional single-visit study, does not determine the effects of dopaminergic medications or shed light on the therapeutic measures.

- The study finds significant correlation of cognition with micrographia based on MMSE testing but does not use detailed cognitive assessment battery.

\section{INTRODUCTION}

Micrographia is a clinical feature commonly associated with Parkinson's disease (PD). The literature, however, reveals a paucity of data on the prevalence and on the clinical characteristics of this potentially disabling disease manifestation. In one study, an overall prevalence of $30 \%$ was observed (at any time during their disease course, with $5 \%$ reporting micrographia as a prodromal symptom. ${ }^{1}$ In questionnaire-based crosssectional studies, the prevalence has ranged from as low as $9 \%^{2}$ to as high as $75 \%$. $^{3}$ Additionally, micrographia has been found to have a high positive likelihood ratio ${ }^{5}$ of being associated with an accurate diagnosis of PD. The phenomenon of micrographia is not restricted to PD but has been reported in Huntington's disease, ${ }^{6}$ amyotrophic lateral 
sclerosis $^{7}$ and lupus $^{8}$ conditions; however, these studies lack adequate number of patients to draw any conclusion on the specificity of the symptom.

Micrographia has been defined as an impairment of a fine motor skill manifesting mainly as a progressive reduction in amplitude during a writing task. Micrographia can manifest in two dimensions. Handwriting may decrease in amplitude as one writes across a single line or manifest as each line gets added with continued writing in a paragraph. ${ }^{9-11}$

In PD, micrographia has been observed to accompany both bradykinesia and hypophonia, ${ }^{2} 912$ and it has been suggested that there is an overlap in the pathophysiology of micrographia and hypophonia. ${ }^{13}$ In this current study, we sought to study PD patients utilizing systematic clinical assessments in order to accomplish the following three aims: (1) to identify the prevalence of micrographia in a large well-characterized PD cohort, (2) to document the clinical profile of micrographia and (3) to determine if a correlation exists between micrographia, bradykinesia or hypophonia.

\section{METHODS}

The study was a single-visit study approved by the local Institutional Review Board, and all subjects gave written informed consent to participate. Subjects with PD were enrolled from a movement disorders clinic located in a veterans adminstration medical centre. PD subjects using United Kingdom Parkinson's Disease Society criteria $^{14}$ were included. All PD patients who presented to the clinic for their regular follow-up visit were approached on a consecutive basis over a period of 2 years, and those who consented to participate were enrolled.

Subjects with neurological conditions like stroke and peripheral neuropathy that could potentially impair the handwriting assessment were excluded. Subjects with possibility of atypical parkinsonism, stroke, neuropathy in hands, $\mathrm{h} / \mathrm{o}$ of significant tremors, dystonia- and levodopa-induced dyskinesias, and those unable to provide informed consent (MMSE <18) were all excluded. Age- and sex-matched control subjects were enrolled from general neurology clinics, including headache, seizures, low back pain and other non-basal ganglia neurological conditions. We specifically sought a non-basal ganglia control group that had a neurological disease in an effort to create a reasonable comparator group. Demographics of PD subjects (age, handedness, language preference), disease duration, L-Dopa dose and disease-specific assessments such as modified Hoehn \& Yahr staging and Unified Parkinson's Disease Rating Scale (UPDRS) were used. All subjects were asked if they had experienced any change in their mental faculties during the course of PD, and Mini Mental Status examination (MMSE) scoring was used during the physical exam. Subjects were studied in the 'ON' medication condition that was defined as being on their regular PD medications and subjectively reporting their typical 'on' response while being examined. Pres- ence of 'micrographia' was ascertained on history. Subjects were asked if they had specifically noted a decrease in size of letters in their handwriting during the writing task. Handwriting was then documented by a bedside clinical handwriting test in both PD and control groups. The handwriting test was designed by us specially for assessment of micrographia and was validated among the control group. In this test, subjects were asked to write the letters ' $p$ ' and ' $d$ ' using lower case in print style and using a standard diameter ball point pen on a lined paper. They were instructed to do this 20 times in two separate rows (figure 1). These 20 trials for each letter were written in blocks, and there were four such blocks consisting of five trials each. Time was not a constraining factor, but subjects were allowed to lift the pen only at the end of each block. A visual model was presented at the beginning of the test, and no practice session for writing was allowed. Auditory cues by the examiner were allowed. For analysis, trials from the first and last block of the letter ' $d$ ' were used, and areas of the 2 blocks were calculated (height $\times$ width). For each block, height was calculated as the maximum

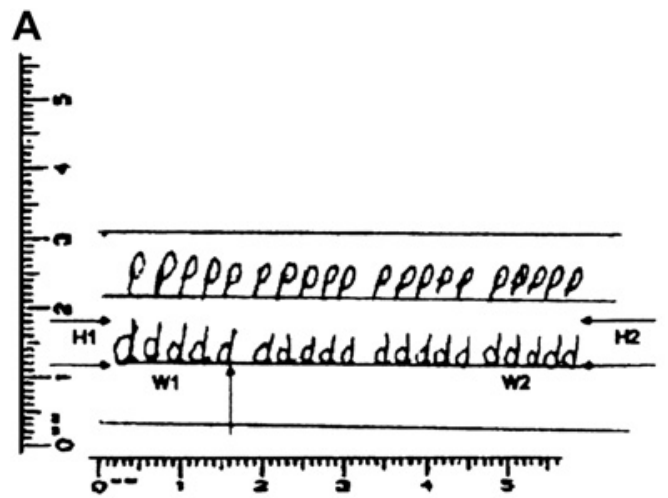

\section{B}

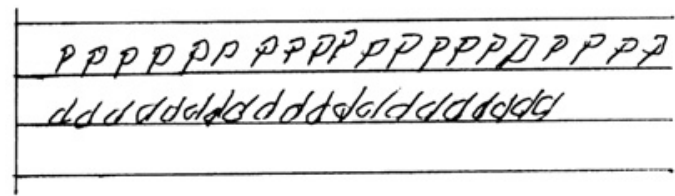

Figure 1 (A) Handwriting sample of subject 1 with PD. The letters ' $p$ ' and ' $d$ ' have been written using lower case in print style, on a lined paper, 20 times in two separate rows. These 20 trials for each letter are written in blocks and there are four such blocks consisting of five trials each. $\mathrm{H} 2$ and $\mathrm{H} 1$ represent maximum vertical stroke in last and first blocks, respectively, and $\mathrm{W} 2$ and $\mathrm{W} 1$ represent respective total distance traveled horizontally for the two blocks; these measurements are for the letters in the second row. Areas for the last $(\mathrm{H} 2 \times \mathrm{W} 2)$ and the first $(\mathrm{H} 1 \times \mathrm{W} 1)$ blocks was calculated and an area drop of $\geq 30 \%$ (0.7) designated as micrographia and a drop of $>50 \%$ as severe micrographia. $\mathrm{H} 2 \times \mathrm{W} 2 / \mathrm{H} 1 \times \mathrm{W} 1<0.7$ consistent with micrographia. $\mathrm{H} 2 \times \mathrm{W} 2 / \mathrm{H} 1 \times \mathrm{W} 1<0.5$ consistent with severe micrographia. (B) Handwriting sample of subject 2 with PD. Second row letters are more crowded than the first, crowding particularly notable in the last few trials. 
vertical stroke achieved, and the width was calculated as the total distance traveled by the pen horizontally. Areas were determined for the last and first blocks, and ratio of these areas was calculated; micrographia was defined as an area drop $\geq 30 \%$; a drop of $>50 \%$ represented severe micrographia (figure 1). The investigators (AWS and SWM) performed this assessment and were not blinded to the two groups, and the method of testing was validated among the neurological controls.

Hypophonia was determined by history based on specific questioning during interview where subjects were asked if they experienced a clear reduction in the volume of their speech. Difficulty in speaking such as stuttering or slurring of words was rejected for the diagnosis of hypophonia. The volume of speech or loudness was documented objectively with a decibel meter. Syllable 'A' was spoken as naturally as possible 10 times at $3 \mathrm{~s}$ intervals in a quiet room. The loudness of speech was recorded with the decibel meter being placed at a set distance of $50 \mathrm{~cm}$ from the mouth. A loudness decline of $\geq 10 \mathrm{~dB}$ between the first and tenth trials was defined as an objective hypophonia (the method also validated in controls).

Bradykinesia was ascertained on history by specifically asking for problems with slowness in movement and UPDRS II questions. Quantitative assessment of bradykinesia was achieved with the help of a finger tap task, Purdue Pegboard testing and a standardized timed motor walk. ${ }^{13} 15$ In the finger tapping task, the subject was required to tap repetitively on a hard surface using the index finger of the right and left hand, done for each side, for $30 \mathrm{~s}$; the number of taps performed in this duration was recorded. The pegboard task involved placement of as many pegs as possible over a $30 \mathrm{~s}$ period and was performed with the right and left hands separately and with both hands simultaneously. For the walking test, subjects walked a distance of $7 \mathrm{~m}$ back and forth as fast as they could, and the time required for walking including turns was recorded. ${ }^{15}$

\section{Statistical analysis}

All statistical analyses were conducted using SAS V.9.2 (SAS Institute Inc.). Data were described with plotting of mean/median for all variables, including age, disease duration, disease severity on Hoehn \& Yahr (H\&Y) staging and on total as well as motor subsection of UPDRS scale, levodopa (L-DOPA) dose, micrographia determined by history and handwriting test analysis, hypophonia by history and by decibel meter recordings, scores on MMSE testing, scores on finger tap task, Purdue Pegboard and timed walking test. $\chi^{2}$ Test was used for comparison of PD and control cohorts with regards to handwriting test and decibel meter readings. Spearman's correlation with respective approximated 95\% CIs based on Fisher's z-transformation was performed to compare all of the above variables with micrographia (figure 2). Cohen's Kappa statistic was used to assess the agreement levels between micrographia and dichotomous variables, such as handwriting scores $(\leq 0.7$ or $>0.7$; a $30 \%$ decline $)$
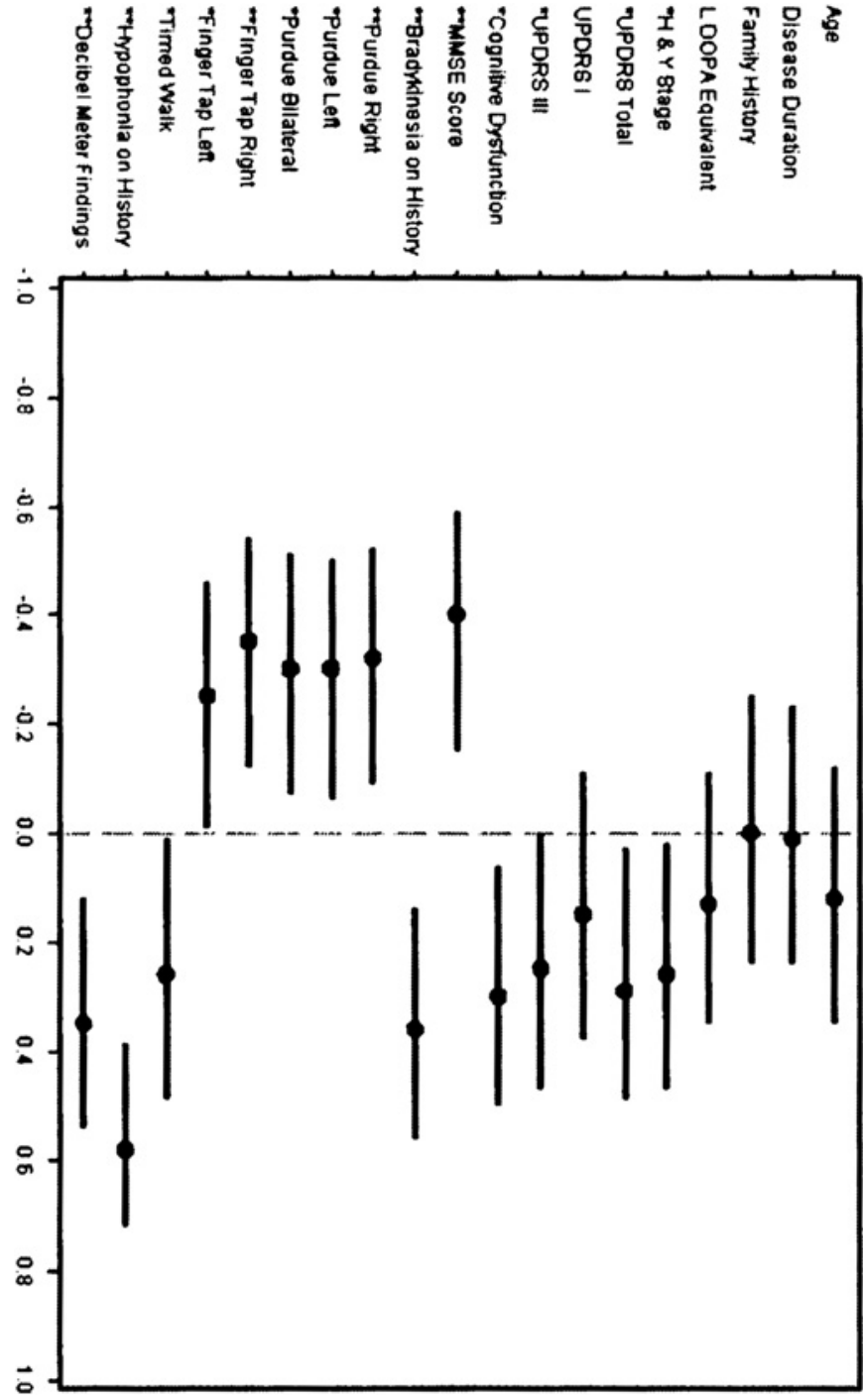

Figure 2 Forest plot used for demonstration of micrographia determined by history and its correlation with demographics, bradykinesia and hypophonia measures. ${ }^{*} p<0.05,{ }^{* *} p \leq 0.01$.

and hypophonia scores $(<10$ or $\geq 10 \mathrm{~dB})$. All results were based on two-sided test with $p$ values $<0.05$ considered statistically significant.

\section{RESULTS}

\section{Demographics}

Sixty-eight PD subjects were enrolled (all were men, mean age $=72.3$ years; mean disease duration $=7.8$ years $)$ (see table 1). Twenty additional subjects were approached; however, they did not consent for participation.

Seven subjects had disease duration of $<3$ years, and six had disease durations $>15$ years. All but two subjects were right handed; one of these two was ambidexterous. In language preference, all except two spoke only one language and that was English. Their levodopa equivalents, UPDRS and MMSE assessments are shown in table 1 . There were no subjects noted to have tremors and dystonia at the time of assessment most likely due to the fact that subjects were studied on medications. 


\begin{tabular}{lc}
\multicolumn{2}{c}{ Table 1 Clinical characteristics of PD subjects } \\
\hline & Mean \pm SD \\
\hline Age in years & $72.3 \pm 9.5$ \\
PD duration in years & $7.8 \pm 5.5$ \\
H\&Y stage & $2 \pm 0.8$ \\
UPDRS total (on score) & $49.3 \pm 18.8$ \\
UPDRS I & $3.1 \pm 2.1$ \\
UPDRS III & $29.1 \pm 9.5$ \\
L-DOPA equivalent in milligram & $766.6 \pm 500.5$ \\
MMSE & $24.8 \pm 2.65$ \\
Purdue Pegboard score & \\
Right hand & $7.7 \pm 2.9$ \\
Left hand & $6.8 \pm 3$ \\
Bilateral assessment & $8.5 \pm 4.1$ \\
Finger tap score & \\
Right hand & $61.7 \pm 27.2$ \\
Left hand & $60 \pm 30.3$ \\
Walk time in seconds & $16.5 \pm 7.1$ \\
\hline H\&Y, Hoehn \& Yahr stage; UPDRS, Unified Parkinson's Disease \\
Rating Scale; MMSE, mini mental status examination; PD, \\
Parkinson's disease.
\end{tabular}

However, mild dyskinesia was observed in two subjects at the time of assessment. In addition, general neurological exam did not reveal presence of neuropathy or stroke like deficits.

All controls enrolled were age matched, were men and like PD subjects were right handed.

\section{Prevalence of micrographia}

Micrographia was present in $63.2 \%$ of the PD cohort (43 subjects) when subjects were asked on history if their handwriting had specifically become small. Nearly $50 \%$ of the PD cohort (35 subjects) demonstrated micrographia when the bedside handwriting test was performed. There was no control subject who reported micrographia on history. On $\chi^{2}$ testing, the control handwriting scores (dichotomized $\leq 0.7$ or $>0.7$; $p=0.0001)$ differed significantly from PD $(p=0.0001)$. The sensitivity for the handwriting test was determined to be $=0.74$ (95\% CI: 0.59 to 0.86 ) and specificity $=0.88$ (95\% CI: 0.68 to 0.97 ). Cohen's $\kappa$ established moderate agreement between micrographia assessment on history and handwriting tests $(0.5,95 \% \mathrm{CI}=0.38$ to 0.77$) ; 15$ PD subjects had a unilateral disease (defined by Hoehn and Yahr Staging scale) with six having a score of 1 and nine having a score of 1.5. Eight of these nine subjects demonstrated micrographia, with the affected side being the dominant right in all patients.

\section{Assessment of hypophonia and Bradykinesia}

Thirty-eight PD subjects (out of 68) reported presence of hypophonia when specifically asked on history. Thirty-six subjects showed a decline of $\geq 10 \mathrm{~dB}$ when decibel meter scores were used for determination. Cohen's $\kappa$ revealed significant correlation between hypophonia assessment on history and objective assessment $(0.85,95 \% \mathrm{CI}=0.72$ to 0.98$)$. On $\chi^{2}$ testing, there was significant difference between decibel meter scores in PD cohort and controls $(p=0.0001)$. There were 54 subjects who reported bradykinesia when specifically asked on history. Their quantitative assessment results are shown in table 1.

\section{Factors affecting micrographia}

In the PD cohort, 43 subjects were found to have micrographia based on history. This group showed significant correlation with overall disease severity and motor impairment (H\&Y, UPDRS total and UPDRS III) and cognitive impairment determined both by history and the MMSE testing $(p=0.02$ and $p=0.002$, respectively; figure 2). It correlated with bradykinesia (determined both by history and quantitative testing) and hypophonia (determined both by history and decibel meter testing). Figure 2 shows the $\mathrm{p}$ values.

Micrographia when identified based on handwriting analysis (35 subjects) showed significant correlation with age $(p=0.02)$, MMSE testing $(p=0.04)$, hypophonia by history $(\mathrm{p}=0.01)$ and bradykinesia determined by Purdue testing for the right hand $(p=0.04)$ and for both hands $(p=0.04)$.

This group with micrographia on handwriting test was further divided into subgroups with mild and severe impairment based on criteria described in the Methods section (area decrease $>30 \%$ is micrographia and area decrease $>50 \%$ defined as severe micrographia). Twentythree subjects had mild micrographia, 12 had severe micrographia and two of these 12 had extreme difficulty in completion of the handwriting test. The subgroup with severe micrographia revealed a significant correlation with H\&Y staging, the UPDRS total score $(p=0.003)$ and the UPDRS III motor score $(p=0.01)$, bradykinesia $(p=0.0001)$ and hypophonia $(p=0.0001)$ determined by history. Severe micrographia also correlated with cognitive impairment assessed by history, MMSE testing and UPDRS I $(\mathrm{p}=0.01)$.

\section{DISCUSSION}

This study offers data on a large cohort of PD and control patients. Micrographia was identified in $63.2 \%$ of the PD cohort when subjects were specifically questioned for micrographia on history and detected in nearly $50 \%$ of the cohort when bedside handwriting test was used for quantitative assessment. Disease severity and cognition were identified as important factors affecting micrographia, and furthermore, there was a strong correlation of micrographia with hypophonia and bradykinesia, suggesting a possible overlap in the pathophysiology.

Previous studies and estimates have been hampered by methodological issues including small sample sizes and lack of objective measures. This was a single-visit study where micrographia was identified by history and was established by a quantitative bedside handwriting test. PD patients with handwriting problems may switch their writing style from cursive to print in order to maintain legibility. For simplicity, we therefore utilized only a print style of writing for the assessment; a hierarchy of tasks including writing in cursive style, words, phrases, sentences and paragraphs was not provided to the 
subjects. Subjects were asked to write letters in a specific and standardized way. The area of writing covered by the specified task was determined, calculated by multiplication of height and distance. It has been observed that handwriting seems to decline along subsequent lines as the writing continues in a paragraph. In this study, we therefore chose to compare the handwriting sample of the second line instead of the first. In a previous study, ${ }^{16}$ micrographia was determined based on the decline of height proportional to an increase in the length of writing. We found a similar decline in height of letters; in addition, we found the letters to be overall smaller and more crowded as the handwriting task continued, which is an observation often reported by clinicians. There was a substantial increase in micrographia when the last and the first blocks in second line were compared.

We found 12 PD subjects with severe micrographia and two out of these 12 could barely complete the handwriting test. The handwriting capacity for these two subjects was noted to be significantly diminished and almost illegible even at the time of signing the consent form and on the item of writing a sentence for the MMSE test. These two subjects were assessed at the time when their dopaminergic medications had begun to wear off. Besides, a possibility of underlying apraxia for writing cannot be ruled out, but we did not specifically test for limb-kinetic or ideomotor apraxia.

Handwriting assessment can be potentially marred by the presence of dystonia, tremors, dyskinesias, history of stroke and peripheral neuropathy affecting the hand. These factors were specifically excluded for the PD subjects though we did not exclude limb apraxia, hand injuries, arthritis of neck and hand joints. These factors should be considered too and excluded for future studies.

The subjects who participated in the study were all men and veterans. They were enrolled from a tertiary care centre, and their handwritings were determined by unblinded raters. These factors may have introduced a selection and assessment bias in the methods.

It has been suggested that L-DOPA may partially improve micrographia, but this notion remains to be verified. L-DOPA seems to improve writing speed more than the $\operatorname{size}^{17}$ and affects the amplitude of the premovement EEG potential (Bereitschaft potential) which is abnormal in PD. ${ }^{18}$ Dopaminergic medication also increases striatal-frontal connectivity between the caudate nucleus and prefrontal cortex during motor timing. ${ }^{19}$ These results suggest that L-DOPA effects on handwriting occur possibly at the level of motor programming. In this study, subjects were studied on medications, and no correlation was found for micrographia and L-DOPA equivalent dose. A study on and off medications is definitely required to provide further insight.

In literature, micrographia has been reported to be more frequent in native than secondary languages, owing to impaired execution of more utilized tasks. ${ }^{20} \mathrm{In}$ our population, most subjects spoke only one language
(English). We had only two veterans who were fluent in more than one language (they knew English and Vietnamese); therefore, in this study, we suspect that the true effects of language on micrographia could not be discerned as the sample size of multilingual subjects was small.

There was also a possible confound of handedness on the clinical manifestation of micrographia, which has been proposed to be more frequent in those with left hemispheric lesions. ${ }^{21}$ In our sample, all subjects except two were right handed (one of them was ambidexterous). Due to the homogeneity in the cohort, the effects of handedness could not be determined. Although handwriting assessment was performed using only the dominant hand, presence of micrographia showed positive correlation with bradykinesia scores determined for both sides (Purdue pegboard and finger tap scores). It would therefore be interesting to determine if handwriting performance was affected bilaterally that was something not focused on in this study.

Presence of micrographia did not reveal any statistical correlation with the overall disease duration though there was a correlation with disease severity determined on $\mathrm{H} \& \mathrm{Y}$ staging $^{22}$ and UPDRS motor assessments. It is also important to note that most subjects were unable to recall the exact onset of timing for handwriting impairment. Thus, a longitudinal follow-up of these patients will be required to determine the effects of disease progression.

The effects of external cues on the task of handwriting in PD are not clear from literature. One study demonstrated micrographia to get better in the presence of both visual as well as auditory cues ${ }^{23}$; in contradistinction, Ondo and Satija ${ }^{16}$ found handwriting to get larger when the subjects performed the handwriting task with eyes closed. We decided to keep the task simple and allowed both visual and auditory cues to be provided during the bedside handwriting test. The handwriting test was conducted on lined paper, and a visual model was presented at the beginning of the task. Auditory announcements for task commencement and task conclusion were made; although unlike the previous study ${ }^{23}$ no specific reminder to keep handwriting big was provided.

It has been observed that there is a significant influence of mental load on micrographia ${ }^{24}$ and increased processing demands within the writing task contribute to reduction in writing size. ${ }^{25}$ In this study, although the mental load was kept at a minimum during the handwriting test and a hierarchy of tasks was not tested, we found those with reports of cognitive impairment on history and also with lower scores on MMSE testing showed a correlation with micrographia. We found that the MMSE scores in this cohort were lower than what one could expect for the H\&Y scores recorded. This was very intriguing, and we think that there could be multiple factors contributing. The study was performed in an older population, we did not record the educational backgrounds of participants, their medication records were not reviewed and a detailed cognitive 
assessment was not performed. Unfortunately, MMSE testing in PD serves only as a screening tool and does not capture all aspects of cognitive functioning.

In this cohort, micrographia revealed a significant correlation with both bradykinesia and hypophonia. Inappropriate scaling of the dynamic muscle force to the movement parameters has been proposed to be one of the underlying mechanisms for bradykinesia ${ }^{26}$ and handwriting issues have been found to be more apparent when a sustained ramp force is required over the duration of a writing stroke. ${ }^{27}$ Similarly, inappropriate scaling of laryngeal muscles during speech that results from a hypometric output sent by motor-premotor cortex is the underlying basis of hypophonia in PD. PET studies have found abnormal patterns of activation in motorpremotor cortex that may be influenced by Lee Silverman Voice therapy, with activation pattern shifting to basal ganglia and insula. ${ }^{28}$ Based on the weight of the evidence, one could hypothesize that micrographia similar to bradykinesia and hypophonia is probably due to a hypometric output driven by motor-premotor cortex with defects in execution of handwriting instructions (inappropriate scaling) and that this may explain the link to bradykinesia and hypophonia.

Future studies of PD-related micrographia should be directed towards functional imaging and electrophysiological assessment of the cerebral cortex and its basal ganglia connections and the on/off effects of dopaminergic medications. This study is the largest of its kind in the area of micrographia and it will help practitioners to understand that the issue is present in about half of all PD patients and that disease severity, cognitive impairment, bradykinesia and hypophonia all seem to be correlated, suggesting an overlap in the pathophysiology.

Acknowledgements We would like to thank Ms Larae Bearden and Ms Kirsten Harper for their help in execution of the project.

Contributors AWS was involved in conception and design, acquisition of data, interpretation of data, drafting the article and final approval of the version; SO was involved in interpretation of data, drafting the article and final approval of the version; MSO, VG and JS were involved in revising the article critically for important intellectual content and final approval of the version to be published. SWM was involved in conception and design, acquisition of data, drafting the article and final approval of the version.

Funding This research received no specific grant from any funding agency in public, commercial or not-for-profit sectors.

Competing interests MSO serves as a consultant for the National Parkinson's Foundation and has received research grants from NIH, NPF, the Michael J. Fox Foundation, the Parkinson's Alliance, Smallwood Foundation and the UF Foundation. MSO has in the past $>24$ months received no support from industry including travel. MSO has received royalties for publications with Demos, Manson and Cambridge (movement disorders books). MSO has participated in CME activities on movement disorders sponsored by the USF CME office, Peer View and by Vanderbilt University. The institution and not MSO receives grants from Medtronic and ANS/St. Jude, and the PI has no financial interest in these grants. MSO has participated as a site $\mathrm{PI}$ and/or co-investigator for several $\mathrm{NIH}$, foundation and industry-sponsored trials over the years but has not received honoraria. VG: employment-Central Arkansas Veterans Health Care System; JS: employment-Central Arkansas Veterans Health Care System; SWM: employment-Central Arkansas Veterans Health Care System.
Provenance and peer review Not commissioned; externally peer reviewed.

Data sharing statement There are no additional data to share.

\section{REFERENCES}

1. McLennan JE, Nakano K, Tyler HR, et al. Micrographia in Parkinson's disease. J Neurol Sci 1972;15:141-52.

2. Ishihara LS, Khaw KT, Luben R, et al. Self-reported parkinsonian symptoms in the EPIC-Norfolk cohort. BMC Neurol 2005;5:15.

3. Jarzebska E. Evaluation of effectiveness of the micrographia's therapy in Parkinson's disease patients. Pol Merkur Lekarski 2006;20:688-90.

4. Duarte J, Claveria LE, de Pedro-Cuesta J, et al. Screening Parkinson's disease: a validated questionnaire of high specificity and sensitivity. Mov Disord 1995;10:643-9.

5. Mutch WJ, Smith WC, Scott RF. A screening and alerting questionnaire for parkinsonism. Neuroepidemiology 1991;10:150-6.

6. Iwasaki Y, Ikeda K, Shindoh T, et al. Micrographia in Huntington's disease. J Neurol Sci 1999;162:106-7.

7. livanainen M, Laaksonen R, Niemi ML, et al. Memory and psychomotor impairment following high-dose interferon treatment in amyotrophic lateral sclerosis. Acta Neurol Scand 1985;72:475-80.

8. Ishihara T, Ozawa T, Otsuki M, et al. Atypical micrographia associated with corticostriatal white matter lesions in systemic lupus erythematosus. J Neurol Neurosurg Psychiatry 2006;77:993-4.

9. Nakamura M, Hamamoto M, Uchida S, et al. A case of micrographia after subcortical infarction: possible involvement of frontal lobe function. Eur J Neurol 2003;10:593-6.

10. Ho SL. Micrographia in Chinese characters. J Neurol Neurosurg Psychiatry 1995;59:471

11. Yoshida T, Yamadori A, Mori E. A case of micrographia with the right hand due to left putaminal infarction. Rinsho Shinkeigaku 1989;29:1149-51.

12. Kuoppamaki M, Rothwell JC, Brown RG, et al. Parkinsonism following bilateral lesions of the globus pallidus: performance on a variety of motor tasks shows similarities with Parkinson's disease. J Neurol Neurosurg Psychiatry 2005;76:482-90.

13. Murray BJ, Llinas R, Caplan LR, et al. Cerebral deep venous thrombosis presenting as acute micrographia and hypophonia Neurology 2000;54:751-3.

14. Hughes AJ, Daniel SE, Kilford L, et al. Accuracy of clinical diagnosis of idiopathic Parkinson's disease: a clinico-pathological study of 100 cases. J Neurol Neurosurg Psychiatry 1992;55:181-4.

15. Defer GL, Widner H, Marie RM, et al. Core assessment program for surgical interventional therapies in Parkinson's disease (CAPSIT-PD). Mov Disord 1999;14:572-84.

16. Ondo WG, Satija $P$. Withdrawal of visual feedback improves micrographia in Parkinson's disease. Mov Disord 2007;22:2130-1.

17. Balas I, Llumiguano C, Doczi TP. Ablative stereotactic surgery improves manual performance time in Parkinson's disease. Parkinsonism Relat Disord 2006;12:223-7.

18. Dick JP, Rothwell JC, Day BL, et al. The Bereitschaftspotential is abnormal in Parkinson's disease. Brain 1989;112:233-44.

19. Jahanshahi M, Jones CR, Zijlmans J, et al. Dopaminergic modulation of striato-frontal connectivity during motor timing in Parkinson's disease. Brain 2010;133:727-45.

20. Yazawa S, Kawasaki S, Ohi T. Is there less micrographia in foreign language in Parkinson's disease? Neurology 2003;61:1817.

21. Marinella MA. Subcortical stroke presenting as micrographia. Am J Emerg Med 2007;25:89-90.

22. Hoehn MM, Yahr MD. Parkinsonism: onset, progression, and mortality. 1967. Neurology 2001;57(10 Suppl. 3):S11-26.

23. Oliveira RM, Gurd JM, Nixon P, et al. Micrographia in Parkinson's disease: the effect of providing external cues. J Neurol Neurosurg Psychiatry 1997;63:429-33.

24. van Gemmert AW, Teulings HL, Stelmach GE. The influence of mental and motor load on handwriting movements in parkinsonian patients. Acta Psychol (Amst) 1998;100:161-75.

25. Van Gemmert AW, Teulings HL, Stelmach GE. Parkinsonian patients reduce their stroke size with increased processing demands. Brain Cogn 2001;47:504-12.

26. Berardelli A, Dick JP, Rothwell JC, et al. Scaling of the size of the firs agonist EMG burst during rapid wrist movements in patients with Parkinson's disease. J Neurol Neurosurg Psychiatry 1986;49:1273-9.

27. Van Gemmert AW, Teulings HL, Contreras-Vidal JL, et al. Parkinson's disease and the control of size and speed in handwriting Neuropsychologia 1999;37:685-94.

28. Liotti M, Ramig LO, Vogel D, et al. Hypophonia in Parkinson's disease: neural correlates of voice treatment revealed by PET. Neurology 2003;60:432-40. 